\title{
Neuropsychophysical optimization by REAC technology in the treatment of: sense of stress and confusion. Psychometric evaluation in a randomized, single blind, sham-controlled naturalistic study
}

This article was published in the following Dove Press journal:

Patient Preference and Adherence

16 March 2012

Number of times this article has been viewed

\author{
Vania Fontani ${ }^{\prime}$ \\ Lucia Aravagli' \\ Matteo Lotti Margotti ${ }^{2}$ \\ Alessandro Castagna' \\ Piero Mannu' \\ Salvatore Rinaldi \\ 'Department of Neuro Psycho \\ Physio Pathology, ${ }^{2}$ Department of \\ Information Technology and Statistical \\ Analysis, Rinaldi Fontani Institute, \\ Florence, Italy
}

Purpose: The aim of this study is to investigate the effects of neuropsychophysical optimization (NPPO) protocol treatment by radioelectric asymmetric conveyer (REAC) technology in the management of sense of stress and confusion (SSC); an analysis of a single cluster of the psychological stress measure (PSM) test.

Patients and methods: The PSM, a self-administered questionnaire, was used to measure psychological stress and SSC for a group of 888 subjects. Data were collected immediately prior to and following the 4-week REAC-NPPO treatment cycle.

Results: This study demonstrates a significant reduction in scores measuring subjective perceptions of stress for subjects treated with one cycle of REAC-NPPO. At the end-point of the study, the number of treated subjects reporting symptoms of stress-related SSC on the PSM test was significantly reduced, whereas there was no difference in sham-treated subjects.

Conclusion: One cycle of REAC-NPPO appears to reduce subjective perceptions of SSC measured by the PSM.

Trial registration: This trial has been registered in the Australian New Zealand Clinical Trials Registry as ACTRN12607000497404.

Keywords: stress disorders, adaptation disorders, REAC, NPPO

\section{Introduction}

The sense of stress and confusion $(\mathrm{SSC})^{1,2}$ is a very common symptom. SSC can afflict anyone at any age ${ }^{3}$ of life, regardless of the type of work they do. ${ }^{4-6}$ Often SSC is not perceived by the affected subjects who, for this reason, suffering the failure or altered perception of their state, can be committed to physical and psychological disorders and/or to implement detrimental behaviors, both to himself and to other people. Specific psychometric tests, such as the psychological stress measure (PSM), in addition to measuring the overall state of stress, allow us to detect the presence of defined stress-related cluster as SSC. Other studies have demonstrated the efficacy of neuropsychophysical optimization (NPPO) treatment protocol by radioelectric asymmetric conveyer (REAC) $)^{7,8}$ in reducing the expression of stress-related symptoms. Since SSC is not a dimensional measure of stress-related complaints, this study aimed to evaluate only the change in the presence of PSM-cluster subjects in SSC as a result of the NPPO or sham treatment, and not the intensity of SSC. ${ }^{9-11}$ The four ${ }^{12}$ sets of 
specific questions of the PSM test were considered valid for identifying SSC in a subject.

\section{Material and methods}

\section{Participants}

This was a naturalistic study, so patients with SSC came unsolicited to our private medical center, the Rinaldi Fontani Institute in Florence, Italy, which is specialized in stressrelated disorders, and were observed in normal clinical practice. During the first visit, each patient was administered the PSM test. The first list of subjects was randomized by a computer using an about 1:4 ratio.

Patients are thus divided into two groups: the real treatment group (RTG), which will be administered the effective therapy, and the sham treatment group (STG). Subjects enrolled in this second group were requested for their availability to participate in a blinded study, where they could receive no effective treatment (sham). Upon acceptance, the administration of effective therapy at the end of any sham treatment was ensured. By using results from the PSM test, we selected from both groups those subjects expressing the SSC cluster: 513 patients in RTG (RTG-SSC) and a second group of 145 subjects (STG-SSC) to whom the sham treatment was administered.

Usually, the patients who attended the Rinaldi Fontani Institute exhibit different types of symptomatology. The main symptoms reported were disorders of sleep, migrant pain, particularly at the cervical and lumbar regions, and decrease of libido. In all subjects, symptoms had been present for several years, and patients had experienced little or no benefit from previous treatments, including drugs, physiotherapy, or any of several psychotherapeutic approaches. The subjects included in the study were asked to avoid taking specific psychotropic drugs. The study was performed in accordance with the Declaration of Helsinki. This trial has been registered in the Australian New Zealand Clinical Trials Registry as ACTRN12607000497404.

\section{Demographic characteristics}

RTG included 688 subjects comprising 401 (58.3\%) females, of average age $42.3 \pm 11.3$ years, and 287 (41.7\%) males, of average age $41.1 \pm 11.4$ years. These patients were treated with active REAC. STG included 200 subjects comprising $123(61.5 \%)$ females, of average age $48.8 \pm 19.4$ years, and $77(38.5 \%)$ males, of average age $45.8 \pm 18.5$ years. These patients were treated with inactive REAC. The PSM test identifies two subgroups of patients who are in SSC cluster: RTG-SSC and STG-SSC. RTG-SSC includes 299 (58.3\%) females, of average age $42.04 \pm 11.3$ years, and $214(41.7 \%)$ males, of average age $41.04 \pm 11.5$ years; STG-SSC includes $90(62.1 \%)$ females, of average age $47.6 \pm 19.2$ years, and $55(37.9 \%)$ males, of average age $45.2 \pm 18.4$ years.

\section{Psychological assessment}

The PSM was developed specifically to detect stress levels in a nonclinical population..$^{9-11}$ The PSM usually consists of a 49-item self-report pencil and paper questionnaire. In this study, we used an electronic version to collect and process the data, and to analyze the results. Patients were asked to answer questions about their psychological stress using a four-point scale to describe the intensity of their condition (very much $=4$, much $=3$, little $=2$, none $=1$ ). To detect the presence of symptoms of SSC, scores were specifically obtained from questions referring to feelings of inadequacy (question 33), not having clear ideas, feelings of confusion, lack of attention and concentration (question 37), perception of a great weight on their shoulders (question 41), and feeling like everything requires a big effort (question 42). Such stress-symptoms items show satisfactory content, criterion, and construct validity for group-level analysis. It is suggested

Table I Compare means

\begin{tabular}{|c|c|c|c|c|c|c|c|}
\hline \multirow{2}{*}{\multicolumn{2}{|c|}{$\begin{array}{l}\text { Sense of stress and } \\
\text { confusion groups }\end{array}$}} & \multicolumn{3}{|c|}{ Treated group } & \multicolumn{3}{|c|}{ Sham-treated group } \\
\hline & & \multirow{2}{*}{$\begin{array}{l}\text { Total pre- } \\
118.53\end{array}$} & \multirow{2}{*}{$\begin{array}{l}\text { Total post- } \\
91.57\end{array}$} & \multirow{2}{*}{$\begin{array}{l}\text { Age } \\
42.33\end{array}$} & \multirow{2}{*}{$\begin{array}{l}\text { Total pre- } \\
119.76\end{array}$} & \multirow{2}{*}{$\begin{array}{l}\text { Total post- } \\
I \mid 2.84\end{array}$} & \multirow{2}{*}{$\begin{array}{l}\text { Age } \\
50.38\end{array}$} \\
\hline 0 & Mean & & & & & & \\
\hline & $\mathrm{n}$ & 175 & 175 & 175 & 55 & 55 & 55 \\
\hline & Standard deviation & 6.620 & 8.176 & 11.305 & 6.75 & 7.76 & 19.7 \\
\hline \multirow[t]{3}{*}{ I } & Mean & 123.90 & 97.52 & 41.62 & 124.17 & 121.83 & 46.68 \\
\hline & $\mathrm{n}$ & 513 & 513 & 513 & 145 & 145 & 145 \\
\hline & Standard deviation & 6.233 & 8.102 & 11.372 & 6.79 & 7.34 & 18.89 \\
\hline \multirow[t]{3}{*}{ Total } & Mean & 122.53 & 96.01 & 41.80 & 122.96 & 122.11 & 47.7 \\
\hline & $\mathrm{n}$ & 688 & 688 & 688 & 200 & 200 & 200 \\
\hline & Standard deviation & 6.747 & 8.520 & 11.35 & 7.04 & 7.45 & 19.14 \\
\hline
\end{tabular}


that the longer scales used to measure psychological stress can be replaced with it in survey research. ${ }^{12}$ In this study, the following types of PSM test results are evaluated: total points and the presence of SSC.

\section{Statistical analysis}

Statistical analysis was performed using SPSS software (v 13; SPSS Inc, Chicago, IL) for the McNemar and Wilcoxon tests and to compare means. The McNemar test analyzed the presence of the SSC symptoms (Table 2), the Wilcoxon test was used to evaluate the significance of PSM total points (Table 3). To evaluate the effectiveness of this treatment the number needed to treat test was used, as an indirect index of the effectiveness of treatments: the smaller the value, the greater the effectiveness of such treatment and vice versa (Table 4). We made a comparison of mean values of the total points of the PSM test correlated with the patients belonging to the SSC cluster, according to their age; this comparison was statistically significant in the sham group, this should not alter the significance of the conclusions of this study (Table 1).

\section{REAC technology and NPPO therapeutic protocol}

The REAC is an innovative technology $\mathrm{y}^{7,8}$ for biostimulation and/or bioenhancement techniques that induces weak radioelectric currents to modulate brain activity. The model used in this study (Convogliatore di Radianza Modulante, ASMED, Italy) is specific for noninvasive brain stimulation techniques. REAC treatment has proved its efficacy in ameliorating several stress-related disorders, ${ }^{13-18}$ depression, ${ }^{18-20}$ anxiety, ${ }^{18,20}$ social anxiety, ${ }^{21}$ agoraphobia, ${ }^{22}$ bipolar disorders, ${ }^{23}$ some forms of dementias, ${ }^{24}$ and impaired motor control. ${ }^{25-27}$ Recently, using specific protocols, REAC tech-

Table 2 McNemar test: psychological stress measure cluster SSC population in treated group and sham-treated group before and after therapy

\begin{tabular}{|c|c|c|c|c|}
\hline \multirow[t]{2}{*}{ Groups } & \multicolumn{3}{|l|}{ Test } & \multirow[t]{2}{*}{ Results } \\
\hline & SSC pre- & SSC & st- & \\
\hline \multirow[t]{3}{*}{$\begin{array}{l}\text { Treated group } \\
(\mathrm{t} 0-\mathrm{t} \mathrm{l})\end{array}$} & & 0 & I & $\begin{array}{l}\text { Asymptotic } \\
\text { significance } 0.000\end{array}$ \\
\hline & 0 & 175 & 0 & Chi-square \\
\hline & I & 282 & 231 & 280.004 \\
\hline \multirow[t]{3}{*}{$\begin{array}{l}\text { Sham-treated } \\
\text { group }(\mathrm{t} 0-\mathrm{t} \mathrm{l})\end{array}$} & & 0 & $I$ & $\begin{array}{l}\text { Asymptotic } \\
\text { significance } 0.610\end{array}$ \\
\hline & 0 & 10 & 45 & Chi-square \\
\hline & I & 51 & 94 & 0.260 \\
\hline
\end{tabular}

Abbreviation: SSC, sense of stress and confusion. nology has demonstrated the chance to induce stem cell pluripotency and differentiation. ${ }^{28}$ NPPO protocol consists of a sequence of seven radiofrequency pulses of 500 milliseconds, applied by touching the metallic tip of the REAC probe onto seven specific auricular points. This protocol consists of 18 sessions, usually administered on alternate days. The REAC procedure is painless, and no adverse effects from its use have been reported.

\section{Results}

Prior to REAC-NPPO, 513 (74.56\%) patients in Group A were positive for SSC. In STG, SSC was detected in 145 (72.5\%) patients. We have noticed that belonging to the cluster SSC is related to the age of the patients (Table 1). Following REAC-NPPO, only 231 of 513 patients (45\%) in RTG still presented symptoms of sense of stress and confusion (McNemar chi-square test 280.004, asymptotic significance 0.000). In STG, SSC was observed in 94 of 145 subjects $(65 \%)$ (Table 2). The Wilcoxon test results are statistically significant for the RTG group (asymptotic significance [two-tailed] $=0.000, \mathrm{Z}=-22.735 ; P<0.005$ ) and not significant for the STG group (asymptotic significance [two-tailed] $=0.361, \mathrm{Z}=-0.914$ ) (Table 3). For the analyzed champion the number needed to treat test confidence interval is between 2.5 and $7.8(1 / 0.40-1 / 0.13)$, number needed to treat test value is equal to 3.83 as demonstrations of effectiveness of the treatment (Table 4).

\section{Discussion}

The current study was designed based upon the results of a previous pilot study that demonstrated the beneficial effects of REAC-NPPO in stress-related disorders, ${ }^{16}$ and was targeted to assess specific stress-related clusters ${ }^{12}$ on the PSM test. ${ }^{9-11}$ Although there is a common knowledge that stress can be the basis for many diseases and disorders, which can also seriously affect the physical and mental health, ${ }^{29}$ the subjective perception of the state of SSC is often denied or underestimated by the patients. In fact, asking all patients who showed the presence of the cluster SSC in the elaboration of the PSM, why they came to our clinic, no one has said that the reason for the consultation was for SSC, but for specific disorders such as migrant pain, particularly at the cervical and lumbar regions, sleep disorders, and decrease of libido. Furthermore, asking specifically whether they thought they suffered from SSC, only a small percentage, about $10 \%$, answered affirmatively, giving it a marginal significance. The lack of perception or underestimation of SSC by the patient can be considered as a risk factor for health status. 
Table 3 Wilcoxon test: psychological stress measure cluster SSC population in RTG and STG before and after therapy

\begin{tabular}{llll}
\hline Groups & Total points & Subjects & Results \\
\hline TG $(\mathrm{n}=688)$ & $122.53 \pm 6.747$ & $\mathrm{n}=5 \mid 3$ & Asymptotic significance (2-tailed) \\
$(\mathrm{t} 0)$ & & 0.000 \\
$\mathrm{Z}=-22.735 P<0.005$ \\
TG $(\mathrm{tl})$ & $\mathrm{n}=23 \mid(45 \%$ of $5 \mid 3)$ & Asymptotic significance $(2$-tailed $)$ \\
STG $(\mathrm{n}=200)$ & $96.0 \mid \pm 8.520$ & $\mathrm{n}=145$ & $0.36 \mid$ \\
$(\mathrm{t} 0)$ & $|22.96 \pm 7.04|$ & & $\mathrm{Z}=-0.9 \mid 4$ \\
STG $(\mathrm{tl})$ & & $\mathrm{n}=94(65 \%$ of $\mid 45)$ & \\
\hline
\end{tabular}

Abbreviations: TG, treated group; STG, sham-treated group; SSC, sense of stress and confusion.

Likewise, the failure to recognize or underestimation of SSC by the physician, can lead to incorrect treatment, which may be of limited effectiveness and/or detrimental. This poses a major, larger question, as to whether truly effective drugs may be available for preventing or treating SSC (an area of enquiry already reviewed).$^{30}$ Basically, we can confirm that there are no drug therapies currently available for preventing or treating SSC, but only to blunt certain symptoms, often with poor therapeutic response and even side effects. The lack of success of drug treatment is not due to ineffectiveness of the drug, but rather to the wrong prescription for nonrecognition or underestimation of the state of SSC by the physician. From these considerations, a question arises: how can a physician help a patient who comes to him for stress-related symptoms, while the patient is not aware of this reality? Probably, it would be helpful that the physician will be able to make a diagnosis of SSC, not only based on the anamnesis, but also using psychometric tests, such as PSM, so that the patient will acquire a greater awareness of his condition. It may also be useful for the physician, with the exception of the acute phases, to avoid an immediate drug prescription, while controlling the symptoms over time. Finally, it might be worthy that the doctor can provide medical treatment, possibly painless, noninvasive, and above all, that does not require the conviction of the patient in the therapeutic success. REAC-NPPO, on the basis of previous

Table 4 Number needed to treat test analysis

\begin{tabular}{llll}
\hline & Present & Absent & Total \\
\hline Treated group & $23 I$ & 457 & 688 \\
Sham-treated group & 139 & 61 & 200 \\
Total & 370 & 518 & 888 \\
Risk of outcome in treated group & & 0.13 \\
Risk of outcome in sham-treated group & & 0.40 \\
Absolute risk reduction & & & 0.26 \\
Relative risk & & 2.86 \\
Relative risk reduction & & 0.65 \\
Number needed to treat & & 3.83 \\
\hline
\end{tabular}

evidence, seems to fulfill the requirements to treat, and probably, prevent the SSC.

\section{Conclusion}

It is documented that electrical currents produced in various ways can lead to a change of the electrical activity of the nervous system and entire body. In fact, electrical currents are tightly entangled with ionic fluxes, and since the REAC equipment induces a circulating current due to an oscillating radiofrequency field, it will also elicit a variation in the ionic fluxes of the whole body. The REAC technology, with its weak radioelectric currents, most likely induces a new favorable tuning of bioelectric activity in the central nervous system, leading to normalization of "stressed" brain function(s). Hence, the REAC-NPPO treatment protocol, based on the current findings and the results from other double-blinded studies, emerges as a valid therapeutic approach to treat stress-related disorders.

\section{Disclosure}

Salvatore Rinaldi and Vania Fontani are the inventors of radioelectric asymmetric conveyer technology.

\section{References}

1. Delgado C. Sense of coherence, spirituality, stress and quality of life in chronic illness. J Nurs Scholarsh. 2007;39(3):229-234.

2. Schuffel W. Adaptation to stress: meaning and sense in medicine. Dtsch Med Wochenschr. 2005;130(3):85-86. German.

3. Wolinsky FD, Wyrwich KW, Kroenke K, Babu AN, Tierney WM. 9-11, personal stress, mental health, and sense of control among older adults. J Gerontol B Psychol Sci Soc Sci. 2003;58(3):S146-S150.

4. Cabarkapa M, Korica V, Rodjenkov S. Personal traits and a sense of job-related stress in a military aviation crew. Vojnosanit Pregl. 2011;68(2):143-149. Serbian.

5. Reilly JE, Fitzpatrick JJ. Perceived stress and sense of belonging in doctor of nursing practice students. J Prof Nurs. 2009;25(2):81-86.

6. Yam BM, Shiu AT. Perceived stress and sense of coherence among critical care nurses in Hong Kong: a pilot study. J Clin Nurs. 2003; 12(1):144-146.

7. Rinaldi S, Fontani V, inventors; Rinaldi S, Fontani V, assignees. Radioelectric asymmetric conveyer for therapeutic use. United States patent. US EP1301241. October 11, 2006. 
8. Rinaldi S, Fontani V, inventors; Rinaldi S, Fontani V, assignees. Radioelectric csymmetric conveyer for therapeutic use. United States patent US 7333859. 2001.

9. Lemyre L, Tessier R. Measuring psychological stress. Concept, model, and measurement instrument in primary care research. Can Fam Physician. 2003;49:1159-1160.

10. Lemyre L, Tessier R. Mesure du stress psychologique (MSP): se sentir stressé. Rev Canad Sci Comport. 1988;20:302-321.

11. Trovato GM, Catalano D, Martines GF, et al. Psychological stress measure in type 2 diabetes. Eur Rev Med Pharmacol Sci. 2006; 10(2):69-74

12. Elo AL, Leppanen A, Jahkola A. Validity of a single-item measure of stress symptoms. Scand J Work Environ Health. 2003;29(6): 444-451.

13. Collodel G, Moretti E, Fontani V, et al. Effect of emotional stress on sperm quality. Indian J Med Res. 2008;128(3):254-261.

14. Fontani V, Rinaldi S, Aravagli L, Mannu P, Castagna A, Margotti ML. Noninvasive radioelectric asymmetric brain stimulation in the treatment of stress-related pain and physical problems: psychometric evaluation in a randomized, single-blind sham-controlled, naturalistic study. Int J Gen Med. 2011;4:681-686.

15. Rinaldi S, Fontani V, Aravagli L, Margotti ML. Psychological and symptomatic stress-related disorders with radio-electric treatment: psychometric evaluation. Stress Health. 2010;26(5):350-358.

16. Rinaldi S, Fontani V, Aravagli L, Mannu P. Psychometric evaluation of a radioelectric auricular treatment for stress related disorders: a double-blinded, sham-controlled controlled pilot study. Health Qual Life Outcomes. 2010;8(1):31.

17. Rinaldi S, Fontani V, Aravagli L, et al. Stress-related psycho-physiological disorders: randomized single blind sham controlled naturalistic study of psychometric evaluation using a radioelectric asymmetric treatment. Health Qual Life Outcomes. 2011;9:54.

18. Rinaldi S, Fontani V, Moretti E, et al. A new approach on stressrelated depression and anxiety: Neuro-Psycho-Physical-Optimization with Radioelectric Asymmetric-Conveyer. Indian J Med Res. 2010; 132:189-194.

19. Mannu P, Rinaldi S, Fontani V, Castagna A, Margotti ML. Radioelectric treatment vs Es-Citalopram in the treatment of panic disorders associated with major depression: an open-label, naturalistic study. Acupunct Electrother Res. 2009;34(3-4):135-149.
20. Olivieri EB, Vecchiato C, Ignaccolo N, et al. Radioelectric brain stimulation in the treatment of generalized anxiety disorder with comorbid major depression in a psychiatric hospital: a pilot study. Neuropsychiatr Dis Treat. 2011;7:449-455.

21. Fontani V, Mannu P, Castagna A, Rinaldi S. Social anxiety disorder: radioelectric asymmetric conveyor brain stimulation versus sertraline. Patient Prefer Adherence. 2011;5:581-586.

22. Mannu P, Rinaldi S, Fontani V, Castagna A, Margotti ML. Noninvasive brain stimulation by radioelectric asymmetric conveyor in the treatment of agoraphobia: open-label, naturalistic study. Patient Prefer Adherence. 2011;5:575-580.

23. Mannu P, Rinaldi S, Fontani V, Castagna A. Long-term treatment of bipolar disorder with a radioelectric asymmetric conveyor. Neuropsychiatr Dis Treat. 2011;7:373-379.

24. Mannu P, Rinaldi S, Fontani V, Castagna A. Radioelectric asymmetric brain stimulation in the treatment of behavioral and psychiatric symptoms in Alzheimer disease. Clin Interv Aging. 2011;6:207-211.

25. Castagna A, Rinaldi S, Fontani V, Mannu P, Margotti ML. Radioelectric asymmetric brain stimulation and lingual apex repositioning in patients with atypical deglutition: a naturalistic, open-label study. J Multidiscip Healthc. 2011;4:209-213.

26. Castagna A, Rinaldi S, Fontani V, Aravagli L, Mannu P, Margotti ML. Does osteoarthritis of the knee also have a psychogenic component? Psycho-emotional treatment with a radio-electric device vs intraarticular injection of sodium hyaluronate: an open-label, naturalistic study. Acupunct Electrother Res. 2010;35(1-2):1-16.

27. Rinaldi S, Fontani V, Castagna A. Brain activity modification produced by a single radioelectric asymmetric brain stimulation pulse: a new tool for neuropsychiatric treatments. Preliminary fMRI study. Neuropsychiatr Dis Treat. 2011;7:649-654.

28. Maioli M, Rinaldi S, Santaniello S, et al. Radio frequency energy loop primes cardiac, neuronal, and skeletal muscle differentiation in mouse embryonic stem cells: a new tool for improving tissue regeneration. Cell Transplant. September 22, 2011. [Epub ahead of print.]

29. Sapolsky RM. Stress, Glucocorticoids, and damage to the nervous system: the current state of confusion. Stress. 1996;1(1):1-19.

30. Christopher PF. Psychological and drug therapies for post-traumatic stress disorder. Psychiatry. 2009;8(8):301-309.
Patient Preference and Adherence

\section{Publish your work in this journal}

Patient Preference and Adherence is an international, peer-reviewed, open access journal focusing on the growing importance of patient preference and adherence throughout the therapeutic continuum. Patient satisfaction, acceptability, quality of life, compliance, persistence and their role in developing new therapeutic modalities and compounds to

\section{Dovepress}

optimize clinical outcomes for existing disease states are major areas of interest. This journal has been accepted for indexing on PubMed Central. The manuscript management system is completely online and includes a very quick and fair peer-review system. Visit http://www.dovepress.com/ testimonials.php to read real quotes from published authors. 